\title{
ARTÉRIA BASILAR EM COELHOS NOVA ZELÂNDIA: ORIGEM, MORFOMETRIA E RAMOS PRINCIPAIS
}

[Basilar artery in New Zealand Rabbit: origin, morphometry and main branches]

\author{
Carlos Augusto dos Santos Sousa ${ }^{1}$, Larissa Torres Ferreira ${ }^{2}$, Stephanie Cardoso da Silva ${ }^{2}$, Marcelo \\ Salvador Gomes ${ }^{3}$ e Marcelo Abidu-Figueiredo ${ }^{4 *}$ \\ ${ }^{1}$ Discente do Programa de Pós-graduação em Biologia Animal, Área de Anatomia Animal da Universidade Federal Rural do \\ Rio de Janeiro, Seropédica, Rio de Janeiro, Brasil. \\ ${ }^{2}$ Discente de Graduação do curso de Medicina Veterinária da Universidade Federal Rural do Rio de Janeiro, Seropédica, Rio \\ de Janeiro, Brasil. \\ ${ }^{3}$ Discente do Programa de Pós-graduação em Medicina Veterinária (Patologia e Ciências Clínicas), Área de Anatomia \\ Animal da Universidade Federal Rural do Rio de Janeiro, Seropédica, Rio de Janeiro, Brasil. \\ ${ }^{4}$ Professor Adjunto do Departamento de Biologia Animal, Área de Anatomia Animal da Universidade Federal Rural Rio de \\ Janeiro, Seropédica, Rio de Janeiro, Brasil.
}

RESUMO - O objetivo desta investigação foi caracterizar a origem, morfometria e ramos principais da artéria basilar em coelhos Nova Zelândia adultos de ambos os sexos. A fixação foi feita através de cânula plástica introduzida na artéria carótida comum com solução de formaldeído a 10\%. Em seguida foram feitas repleções vasculares com solução aquosa de Petrolátex S65 corado com pigmento Suvinil vermelho. Procedeu-se a craniotomia, remoção dos encéfalos e dissecção para a observação da origem e ramificações principais da artéria basilar. A média e erro padrão do comprimento da artéria basilar foram de 1,293 cm $\pm 0,024$ nas fêmeas e 1,227 $\mathrm{cm} \pm 0,025$ nos machos. Em todos os coelhos dissecados, a artéria basilar se originou da anastomose das artérias vertebrais, direita e esquerda, emitindo a artéria cerebelar caudal, ramos variados para bulbo e ponte, ramos terminais e artérias cerebelares rostrais.

Palavras-Chave: Cérebro; lagomorfa; vascularização.

\begin{abstract}
The aim of this study was to describe the origin, morphometry and main branches of the basilar artery, in adult New Zealand rabbits of both sexes. The setting was made by plastic tube inserted into the common carotid artery with $10 \%$ formaldehyde solution. After fixed and performing the injections with latex solution stained with pigment, the brains were removed after craniotomy and dissected to identify the origin and main branches of the basilar arteries. Mean and standard error of the basilar artery length were $1.293 \mathrm{~cm} \pm 0.024$ in females and $1.227 \mathrm{~cm} \pm 0.025$ in males. In all rabbits dissected, the basilar artery stemmed from the anastomosis of vertebral arteries, right and left, giving rise to the caudal cerebellar artery, varied branches to bulb and pons, terminal branches, and rostral cerebellar arteries.
\end{abstract}

Keywords: brain; lagomorpha; vascularization.

\footnotetext{
* Autor para correspondência. E-mail: marceloabidu@ gmail.com Recebido: 22 de dezembro de 2015.

Aceito para publicação: 15 de fevereiro de 2016.
} 


\section{INTRODUÇÃO}

O estudo morfofuncional do sistema nervoso central em vertebrados demonstra aspectos relevantes que ainda precisam ser explorados, em especial àqueles relativos ao suprimento sanguíneo para o encéfalo (Cunha et al., 2001).

As doenças que acometem o sistema nervoso central dos animais domésticos e silvestres representam grande parcela das afecções observadas na prática clinico-cirúrgica desses animais; e podem causar deficiência de irrigação e consequente lesão isquêmica (Meneses, 2011).

A abordagem cirúrgica desta região requer um conhecimento anatômico preciso, não só da habitual morfologia, mas também das variações das diversas estruturas que ali se encontram.

O estudo das artérias responsáveis pela irrigação do encéfalo nos animais domésticos e silvestres considerando-se os aspectos filogenéticos, ainda é motivo de interesse na pesquisa biomédica devido à grande variabilidade dos arranjos apresentados pelas artérias formadoras do "círculo de Willis" (De Vriese, 1905; Testut; 1911; Barone \& Bortolami 2004; Prada, 2014), embora esses diferentes aspectos morfológicos não representem necessariamente parâmetros evolutivos (De Vriese, 1905). Dessa maneira, acredita-se que o comportamento das artérias encefálicas, comparativamente entre as diferentes espécies, exibe um modelo básico ao qual são acrescentadas modificações relativas aos diferentes grupos de animais (De Vriese, 1905; Testut, 1911).

O uso de animais como modelo experimental acontece há muito tempo e tem possibilitado muitos avanços na pesquisa biomédica, pois permitem controlar numerosas variáveis que não podem ser obtidas em estudos com seres humanos, além de contribuir com informações que propiciem melhorias no manejo e na sanidade dos animais utilizados.

Dentre os modelos experimentais mais usados podemos destacar o suíno em patologias do sistema nervoso e urogenital (Pereira-Sampaio et al., 2004), carnívoros em patologias osteoarticulares e condições de septicemia, roedores e coelhos com testes de novas drogas e patologias relacionadas ao sistema urogenital (Maia et al., 2006). O coelho também tem sido bastante utilizado como modelo experimental em cranioplastia, bem como treinamento em cirurgias estereotáxicas destinadas a ressecções, punções e biópsias para diagnóstico em tumores de diferentes linhagens celulares, que precisam de abordagem terapêutica diferenciada (Zagzag et al., 1998).
Apesar disto, valores morfométricos de referência e vascularização do encéfalo de coelhos ainda são escassos na literatura, necessitando de maior investigação. $O$ presente trabalho tem como objetivo caracterizar a origem, morfometria e ramos principais da artéria basilar em coelhos de ambos os sexos subsidiando a pesquisa aplicada em trabalhos correlatos.

\section{MATERIAL E MÉTODOS}

Esta pesquisa faz parte do projeto intitulado Anatomia Topográfica e Sistêmica do Coelho: estudo destinado a pratica cirúrgica, radiológica e experimental, que foi submetido e aprovado pelo comitê de ética e pesquisa da Universidade Federal Rural do Rio de Janeiro, de número 23083.002379/2007-08.

Os estudos de dissecção anatômica foram realizados em 44 coelhos, 21 machos e 23 fêmeas, pesando em média $2,5 \mathrm{~kg}$. Todos os animais utilizados faziam parte das aulas práticas das disciplinas de Cirurgia e foram cedidos à área de Anatomia

Animal.

Os animais já haviam sido submetidos à eutanásia, com administração de superdosagem de tiopental sódico.

Foram previamente tricotomizados, identificados e posicionados em decúbito lateral direito. Em seguida, o tórax foi aberto e dissecado para evidenciação da aorta torácica, que foi canulada. Desse modo, o sistema arterial foi "lavado" com solução fisiológica de $\mathrm{NaCl}$ a $0,9 \%$, sendo realizado a seguir a fixação com solução de formaldeído a $10 \%$, conforme técnica anatômica utilizada na rotina do laboratório. Em seguida, injetou-se pela cânula solução aquosa (diluição 1:1) de Petrolátex S-65 (Refinaria Duque de Caxias-REDUC- da Petrobrás, Duque de Caxias-RJ) misturado ao corante (Suvinil xadrez ${ }^{\circledR}$ ).

Em seguida os cadáveres foram imersos em caixas de polietileno de baixa densidade de capacidade de 500 litros contendo solução de formaldeído a $10 \%$ para a finalização do processo de fixação e polimerização do látex. Decorridos sete dias da injeção do látex, os cadáveres foram lavados em água corrente e dissecados.

Para abertura dos crânios, remoção dos encéfalos e dissecação dos vasos da base, foram utilizados instrumentais cirúrgicos de rotina e fórceps odontológico. Todas as mensurações lineares foram realizadas pelo mesmo observador, utilizando um paquímetro digital da marca Eda. A artéria basilar foi dissecada com o objetivo de avaliar sua origem, 
medida linear, e variações numéricas, topográficas e antiméricas das ramificações principais (Figura 1). Foi considerado como ponto de referência de início, a convergência das artérias vertebrais direita e esquerda e o fim a última ramificação da artéria basilar. Os vasos foram denominados de acordo com a nomenclatura anatômica veterinária (Schaller, 1999).

A média e o erro padrão da média do comprimento da artéria basilar foi comparada entre os sexos; a média e o erro padrão da média do número de ramificações principais foi comparada entre os sexos e antímeros. As variáveis foram comparadas através do Teste $t$ não pareado, considerando $\mathrm{p}<$
0,05 como significativo. Toda a análise estatística foi realizada no Software Graphpad Prism 5.

\section{RESULTADOS}

A média mais erro padrão do comprimento da artéria basilar foi de 1,227 $\pm 0,025$ nos machos e $1,293 \pm 0,024(\mathrm{p}=0,069)$ nas fêmeas. Tanto nos machos quanto nas fêmeas, as artérias vertebral direita e esquerda, foram as responsáveis pela formação da artéria basilar. Apresentou-se única em $23(100 \%)$ fêmeas, e em 20 (95\%) machos; e em apenas um (5\%) macho se apresentou dupla. Em ambos os sexos, a artéria basilar seguiu de forma retilínea em direção à base do encéfalo (Figura 1).

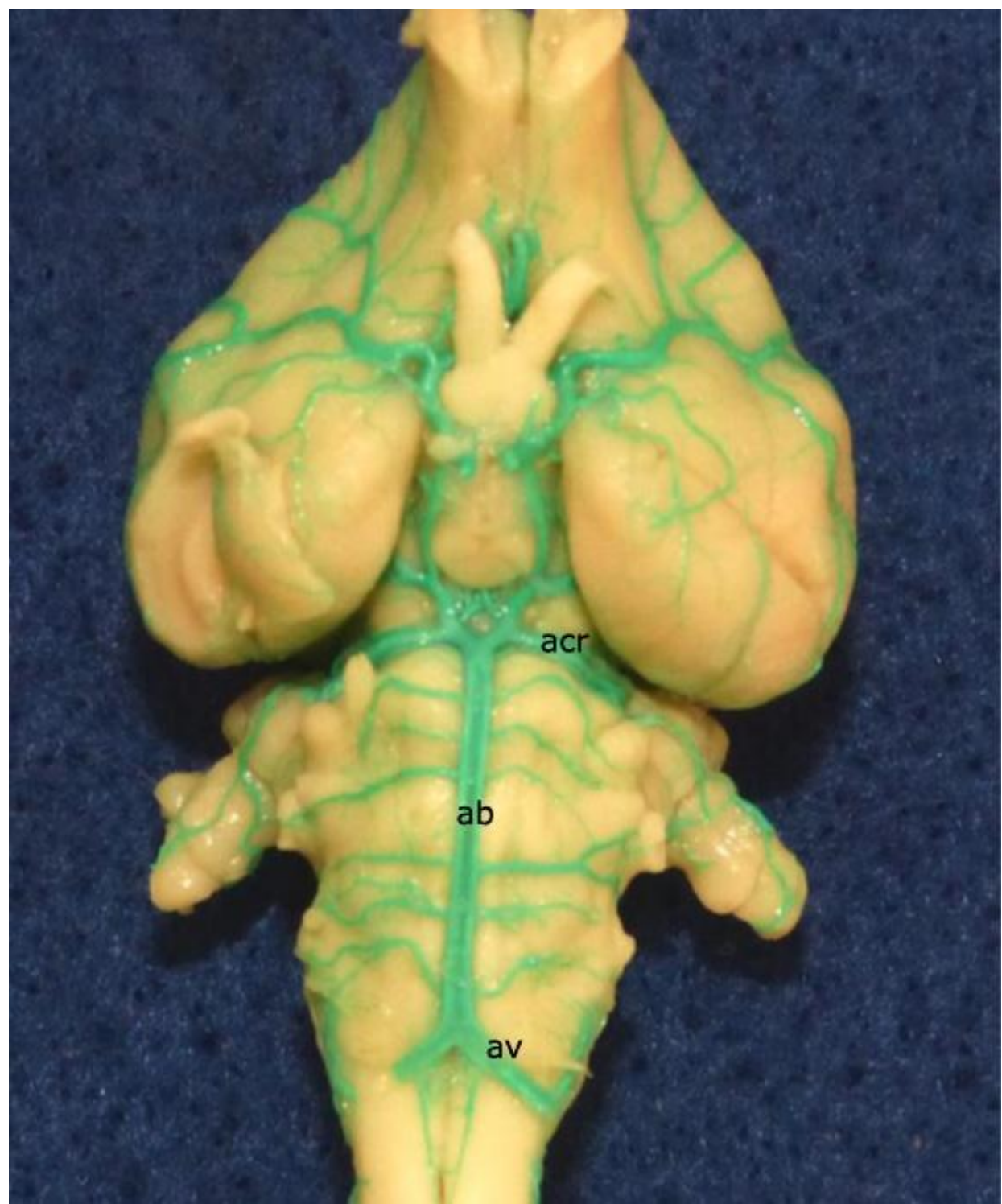

Figura 1. Fotomacrografia da vista ventral do encéfalo mostrando as ramificações principais da arteria basilar: av - artéria vertebral, ab - artéria basilar, acr - artéria cerebelar rostral

A artéria basilar emitiu como primeiro ramo a artéria cerebelar caudal única em ambos os antímeros e em ambos os sexos. Este vaso apresentou simetria de posição em relação aos antímeros em 17 (80,90\%) machos e 17 (73, 90\%) fêmeas e consequentemente assimetria no qual foram observados vasos mais craniais ou caudais ao seu correspondente no antimero oposto em quatro 
$(19,10 \%)$ machos e seis $(26,10 \%)$ fêmeas. Em seguida a artéria basilar emitiu número variado de ramos para bulbo e ponte em ambos os antímeros em machos e fêmeas. Nos machos a média, mais erro padrão da média do número de ramos para o antímero direito foi de $5,429 \pm 0,2723$ e para o esquerdo de $5,190 \pm 0,2140(\mathrm{p}=0,4958)$. Nas fêmeas a média, mais erro padrão da média do número de ramos para o antímero direito foi de $5,522 \pm 0,2424$ e para o esquerdo de $5,609 \pm 0,1859$ $(\mathrm{p}=0,7772)$.

No ponto de bifurcação, a artéria basilar em sua porção rostral emitiu ramos para ambos os antímeros denominados de ramos terminais da artéria basilar em direção ao mesencéfalo.

A artéria cerebelar rostral se originou do ramo terminal da artéria basilar em $17(80,95 \%)$ machos e $21(91,30 \%)$ fêmeas e da artéria basilar, na altura da ponte em quatro $(19,04 \%)$ machos e uma $(4,34$ $\%$ ) fêmea, todos de forma simétrica em ambos os antímeros. Em apenas uma (4,34\%) fêmea, a artéria cerebelar rostral esquerda se originou da artéria basilar na altura da ponte enquanto que a correspondente direita se originou do ramo terminal da artéria basilar.

A artéria cerebelar rostral se apresentou de forma simétrica em $19(90,47 \%)$ machos e $21(91,30 \%)$ fêmeas. A assimetria foi observada em dois $(9,53 \%)$ machos e duas $(8,7 \%)$ fêmeas. Em relação ao número, a artéria cerebelar rostral se apresentou única em ambos os antímeros em 13(61,90\%) machos e $12(52,17 \%)$ fêmeas, dupla em ambos os antímeros em um $(4,76 \%)$ macho e quatro $(17,39 \%)$ fêmeas, dupla no antímero esquerdo e única no direito em quatro $(19,4 \%)$ machos apenas, dupla no antímero direito e única no esquerdo em três $(14,28 \%)$ machos e sete $(30,43 \%)$ fêmeas.

\section{DISCUSSÃO}

Não houve diferença na medida do comprimento da artéria basilar entre o sexo dos coelhos estudados (p $=0,069)$. Na literatura consultada não foram observados valores correspondentes à medida desse vaso.

A artéria basilar, tanto nos machos quanto nas fêmeas, formou-se a partir da anastomose das artérias vertebrais, direita e esquerda semelhante ao observado no gato (Lima et al., 2006; Gomes et al., 2012a). Resultados diferentes foram obtidos por Jewell (1952) em cães, onde a artéria vertebral se juntava à artéria espinhal ventral na superfície ventral da medula espinhal, após receber anastomose das artérias occipitais originando rostralmente a artéria basilar. De La Torre et al. (1962), entretanto, relataram em cães que esta artéria era formada pela união dos ramos dorsais direito e esquerdo do ramo occipito-vertebral, semelhante aos resultados obtidos por Gillilan (1976), que também usou o cão e o gato. Diferente dos autores acima, Pais et al. (2009) observaram que, em todos os cães analisados, as artérias vertebrais direita e esquerda foram as responsáveis pela formação da artéria basilar, corroborando com os resultados da presente investigação.

Em estudo com fetos de bovinos azebuados (Melo \& Prada, 1998) e com fetos de búfalos sem raça definida (Faria \& Prada, 2001), a artéria basilar se apresentou como um vaso ímpar resultante da convergência dos ramos caudais, direito e esquerdo, da artéria carótida; diferente do observado por Ocal et al. (1999), em camelo (Camelus dromedrarius) e Brudnicki (2000), em caprinos, onde em ambos os casos a artéria basilar era formada pela anastomose das artérias vertebrais e artéria espinhal ventral. Os resultados acima diferem dos obtidos na presente investigação, onde a artéria basilar se formou exclusivamente a partir da união das artérias vertebral direita e esquerda.

Almeida \& Campos (2011) observaram que no jacaré do papo amarelo (Caiman latirostris) a artéria basilar era formada a partir da anastomose das partes convergentes do ramo caudal da artéria carótida interna, arranjo semelhante ao observado por Nazer \& Campos (2011), no cérebro de avestruz, onde a artéria basilar apresentou-se como um vaso único em $80 \%$, de forma dupla em $13,3 \%$ e em forma de ilha em $6,7 \%$ das amostras, diferente dos resultados da presente pesquisa no que se refere à formação da artéria basilar; entretanto se assemelha aos resultados dos autores supra citados, pois em coelhos também houve predomínio da forma única.

Variações na artéria basilar foram descritas por Paiva-de-Souza et al. (2010), em equinos mestiços com a frequência variável da artéria caroticobasilar, uma anastomose entre a artéria carótida interna e a artéria basilar observada na face ventral do tronco encefálico. Esta variação não foi observada em coelhos da presente pesquisa, semelhante ao observado em gatos (Gomes et al., 2012a).

Depedrini \& Campos (2003) observaram que na raposa (Dusicyon gymnocercus), a artéria basilar originou-se da artéria vertebral direita e esquerda e pela bifurcação terminal rostral da artéria espinhal ventral, entretanto, de acordo com Wiland (1966), a artéria basilar na raposa forma-se através da união dos ramos da artéria vertebral e occipital. Em ambos os autores acima citados, os resultados obtidos diferem dos verifica dos na presente investigação. 
Em estudo realizado com roedores da espécie Hystrix cristata e Spalax leucodon, Aydin et al. (2005; 2008), respectivamente, observaram que em ambas as espécies a artéria basilar originou se da fusão das artérias vertebral direita e esquerda; este mesmo arranjo foi observado por Reckziegel et al. (2001), em estudo realizado em capivaras (Hydrochoerus hydrochoeris) e também nos resultados obtidos na presente investigação.

Em equinos Puro Sangue Inglês, Campos et al. (2003) observaram que a artéria basilar formou-se a partir da convergência das artérias occipitais direita e esquerda, diferente do que foi observado na presente pesquisa.

Lima et al. (2005), em estudo realizado com encéfalos de suínos da linhagem Camborough 22, Ferreira \& Prada (2005), em suínos sem raça definida, e Oliveira \& Campos (2005), no javali (Sus scrofa scrofa), observaram que a rede admirável epidural caudal e ainda a artéria espinhal ventral foram as responsáveis pela formação da artéria basilar, diferente dos resultados obtidos na presente investigação.

Em chinchilas (Chinchilla lanígera), Araújo \& Campos (2005) observaram que em 96,7\% a artéria basilar formou-se pela convergência das artérias vertebral direita e esquerda, enquanto que, em $3,3 \%$, foi formada pela artéria carótida interna esquerda. Os resultados desses autores, apesar de não estarem expressos separados pelo sexo, assemelham-se, em parte, com os do presente estudo, pois em $100 \%$ dos animais dissecados as artérias vertebrais, direita e esquerda, foram responsáveis pela formação da artéria basilar.

Azambuja (2006) observou que no ratão do banhado (Myocastor ciypus) a artéria basilar formava-se a partir da anastomose dos ramos terminais das artérias vertebral direita e esquerda, semelhante ao que fora observado em coelhos na presente investigação, e aos resultados de Ferreira \& Prada (2001), em estudo sobre os vasos da base do encéfalo do macaco -prego (Cebus apella), Barreiro et al. (2012), em estudo realizado em quatis, e Lima et al. (2013), em tamanduás-mirim.

Em todos os animais dissecados, a artéria basilar emitiu como ramos principais; a artéria cerebelar caudal que se apresentou de forma simétrica e assimétrica em relação ao antímero, seguida de ramos variados em número, e de menor calibre para ponte e bulbo e como último ramo, as artérias cerebelares rostrais, direita e esquerda. Esses resultados assemelham-se aos obtidos por Lima et al. (2006) e Gomes et al. (2012a), para gatos, e Alcântara \& Prada (1996a; 1996b), para o cão. Entretanto, Barreiro et al. (2012) e Lima et al.
(2013) observaram a presença da artéria cerebelar média, vaso este que não foi observado em coelhos.

\section{CONCLUSÃO}

Não existe diferença no comprimento da artéria basilar entre os sexos. A artéria basilar se formou a partir da convergência das artérias vertebrais direita e esquerda em todos os animais. A artéria basilar, em todos os animais dissecados, emitiu como ramos principais; a artéria cerebelar caudal que apresentou, mais frequentemente, simetria de posição em relação ao antímero; seguida de número variado de ramos e de menor calibre para ponte e bulbo e como último ramo, as artérias cerebelares rostrais, direita e esquerda.

\section{AGRADECIMENTOS}

Fundação de Amparo à Pesquisa do Estado do Rio de Janeiro (FAPERJ), Conselho Nacional de Desenvolvimento Científico e Tecnológico (CNPq).

\section{REFERÊNCIAS}

ALCÂNTARA, M. A.; PRADA, I. L. S. Arteries of basis of encephalon in dogs (Canis familiaris, Linnaeus, 1758). I. Anatomical study of sources and behavior. Brazilian Journal of Veterinary Research and Animal Science, São Paulo, v. 33, n. 2, p. 67-71, 1996a.

ALCÂNTARA, M. A.; PRADA, I. L. S. Arteries of basis of encephalon in dogs (Canis familiaris, Linnaeus, 1758). II. Formation and behavior of the encephalon arterial circuit. Brazilian Journal of Veterinary Research and Animal Science, São Paulo, v. 33, n. 2, p. 72-76, 1996 b.

ALMEIDA, L.; CAMPOS, R. A. Systematic study of the brain base arteries in broad-snouted caiman (Caiman latiros tris). Journal of Morphological Science, São Paulo, v. 28, n. 1, p. 62-68, 2011

ARAÚJO, A. P. C.; CAMPOS, R. A. Systematic study of the brain base arteries and their blood supply sources in the chin chilla (Chinchilla lanigera, Molina, 1782). Brazilian Journal of Morphological Science, São Paulo, v. 22, n. 4, p. 221-232, 2005.

AYDIN, A.; DINC, G.; KARAN, M.; OZDEMIR, D.; YILMAZ, $\mathrm{S}$. The morphology of circulus arteriosus cerebri in the porcupine (Hystrix cristata). Veterinarni Medicina, Prague, v. 50, n. 3 , p. $131-135,2005$.

AYDIN, A.; ILGÜN, R.; OZKAN, Z. E.; YILMAZ, S. Morphological investigations on the circulus arteriosus cerebri in mole-rats (Spalax leucodon). Anatomia Histologia Embryologia, Berlin, v. 37, n. 3, p. 219-222, 2008

AZAMBUJA, R. C. Sistematização das artérias da base do encéfalo e suas fontes de suprimento sanguíneo em nutria (Myocastor coypus). 2006. 150 f. Dissertação (Mestrado em Ciências Veterinárias) - Universidade Federal do Rio Grande do Sul, Porto Alegre. 2006.

BARONE, R. \& BORTOLAMI, R. Anatomie comparée des Mammifères domestiques Tome 6: Neurologie I. Système nerveux central. Paris, Vigot Frères éditeurs, 652 p, 2004 
BARREIRO, J. R.; CARVALHO, A. F.; FRANCIOLLI, A. L. R.; FERREIRA, G. J. B. C.; FERREIRA, J. R.; AM BRÓSIO, C. E.; MIGLINO, M. A. Morfologia dos vasos da base do encéfalo do quati (Nasua nasua). Pesquisa Veterinária Brasileira, Rio de Janeiro, v. 32, n. 6, p. 567-572, 2012.

BRUDNICKI, W. Basilar arteries of the brain in domestic goat (Capra hircus). Electronic Journal of Polish Agricultural Universities, Cracow, v. 3, n. 1, p. 1-4, 2000.

CAMPOS, A; DOS SANTOS, D; DOS SANTOS JUNIOR, I; PRADA, I. S. Artérias da base do encéfalo de eqüinos. Sistema occi pito-basilar. Brazilian Journal of Veterinary Research and Animal Science, São Paulo, v. 40, n. 1 p. 107- 117, 2003.

CUNHA IP, FAGUNDES GM, SCAFF RM, XIKOTA JC, HEINZEN RPS, MARIANO PA, ROSÁRIO SRP (2001). Comportamento anatômico da artéria cerebelar média no cão (Canis familiaris - Linnaeus, 1758). Veterinária Notícias, Uberlândia, 7, 13-22.

DE LA TORRE, E.; MICHELL, O. C.; NETSKY, M. A. Anatomic and angiographic studies of de vertebral basilar system in dog. American Journal of Anatomy, Philadelphia, v. 110, n. 2, p. $187198,1962$.

DE VRIESE, B. Sur la signification morphologique des artères cerebrales. Archives de Biologie, v. 21, p. 357-457, 1905.

DEPEDRINI, J. S.; CAMPOS, R. A systematic study of the brain base arteries in the pampas fox (Dusicyon gymnocercus). Brazilian Journal of Morphological Science, São Paulo, v. 20, n. 3, p. $181188,2003$.

FARIA, M. M. M. D.; PRADA, I. L. S. Anatomical behavior of the basilar artery of buffalo fetuses (Bubalus bubalis, Linnaeus, 1758). Revista Brasileira de Saúde e Produção Animal, Salvador, v. 1, n. 2, p. 54-60, 2001

FERREIRA, C. G.; PRADA, I. L. S. O circuito arterial da base do encéfalo em suínos (Sus scrofa domesticus, Linnaeus, 1758), formação e comportamento. Brazilian Journal of Veterinary Research and Animal Science, São Paulo, v. 42, n. 1, p. 53-60, 2005.

FERREIRA, J. R.; PRADA, I. L. S. Nomenclatura proposta para denominar as artérias da base do encéfalo do macaco-prego (Cebus apella, Linnaeus, 1766). Acta Scientiarum, Maringá, v. 23, n. 2, p. 635-643, 2001.

GILLILAN, L. A. Extra and intra-cranial blood supply to brains of dog and cat. American Journal of Anatomy, Philadelphia, v. 146, n. 3, p. 237-254, 1976

GOMES, M. S.; HERNANDEZ, J. M. F.; ALONSO, L. S.; ABIDU-FIGUEIREDO, M. Morfologia e ramos principais da artéria basilar em gatos. Revista Brasileira de Medicina Veterinária, Rio de Janeiro, v. 34, n. 3, p. 206-212, 2012a.

JEWELL, P. A. The anastomoses between internal and external carotid circulation in the dog. Journal of Anatomy, London, v. 86 , n. 2 , p. $83-94,1952$

LIMA, E. M. M.; PRADA, I. L. S.; CARNEIRO E SILVA, F. O.; SEVERINO, R. S.; QUAGLIATTO, A. L.; DRUMMOND, S. S.; RODRIGUES, G. S. Estudo anatômico das artérias da base do encéfalo em gatos. Ars Veterinária, São Paulo, v. 22, n. 1, p. $1-7,2006$.

LIMA, E. M. M.; SEVERINO, R. S.; CARNEIRO E SILVA, F. O.; DRUMMOND, S. S.; BOMBONATO, P. P.; CAMPOS, D. B.; RODRIGUES, G. S. Artérias da base do encéfalo do suíno da linhagem camborough 22. Bioscience Journal, Uberlândia, v. 21, n. 2 , p. $137-147,2005$.
LIMA, A. R.; PEREIRA, L. C.; BRANCO, E. Anatomia do circuito arterial do encéfalo em tamanduá-mirim. Ciência Rural, Santa Maria, v. 43, n. 2, p. 277-282, 2013.

MAIA, R. S.; ABIDU-FIGUEIREDO, M.; BABINSKI, M. A.; CHAGAS, M. A.; COSTA, W. S.; SAMPAIO, F. J. B. Concentration of elastic system fibers in the corpus cavernosum, corpus spongiosum, and tunica albuginea in the rabbits penis. International Journal of Impotence Research, London, v. 18, n. 2 , p. $121-125,2006$

MELO, A. P. F.; PRADA, I. L. S. Anatomical study of arteries of the base of encephalon in fetuses of zebu crossed bovines. Brazilian Journal of Morphological Science, São Paulo, v. 15, n. 2, p. 143149, 1998.

MENESES, S. M. Neuroanatomia aplicada. 3. ed. Rio de Janeiro: Guanabara Koogan, 2011. 368 p

NAZER, M. B.; CAMPOS, R. Systematization of the brain base arteries in ostrich (Struthio camelus). Journal of Morphological Science, São Paulo, v. 28, n. 4, p. 268-274, 2011.

OCAL, M. K.; ERDEN, H.; OGUT, I.; KARA, M. E. A Quantitative study of the circulus arteriosus cerebri of the camel (Camelus dromedarius).Anatomia Histologia Embryologia, Berlin, v. 28, n. 5-6, p. 271-272, 1999.

OLIVEIRA, J. C. D.; CAMPOS, R. A SYSTEMATIC STUDY OF BRAIN BASE ARTERIES IN THE WILD BOAR (SUS SCROFA SCROFA). ANATOMIA HISTOLOGIA EMBRYOLOGIA, BERLIN, V. 34, N. 4, P. 232-239, 2005. PAIS, D.; ARANTES, M.; CASAL, D.; CASIMIRO, M.; O'NEILL, J. G. Brain stem arteries in Canis familiaris implications in experimental procedures. Brazilian Journal of Morphological Science, São Paulo, v. 26, n. 1, p. 8-11, 2009.

PAIVA-DE-SOUZA, A. V.; XAVIER-SILVA, B.; ANTUNES, M. S.; HERNANDEZ, J. M. F.; SCHERER, P. O.; ABIDUFIGUEIREDO, M. Frequência da artéria caroticobasilar em equinos mestiços: estudo anatômico destinado à pesquisa experimental $\mathrm{e}$ ao diagnóstico por imagem. Pesquisa Veterinária Brasileira, Rio de Janeiro, v. 30, n. 8, p. 685-688, 2010.

PEREIRA-SAMPAIO, M. A.; FAVORITO, L. A.; SAMPAIO, F. J. B. Pig kidney: anatomical relationships between the intrarenal arteries and the kidney collecting system. Applied study for urological research and surgical training. Journal of Urology, Baltimore, v. 172, n. 5, p. 2077-2081, 2004.

PRADA, I. L. S. Neuroanatomia Funcional em Medicina Veterinária com correlações clínicas. $1^{\mathrm{a}}$ ed. Editora Terra Molhada, 616 p, 2014

RECKZIEGEL, S. H.; LINDEMANN, T.; CAMPOS, R. A. Systematic study of the brain base arteries in capybara (Hydrochoerus hydrochaeris). Brazilian Journal of Morphological Science, São Paulo, v. 18, n. 2, p. 104-110, 2001.

SCHALLER, O. Nomenclatura anatômica veterinária ilustrada. São Paulo: Manole, 1999. 641 p.

TESTUT, L. Traité de anatomie humaine. 6. ed. Paris: Octave Doin, 1911.

WILAND, C. The basilar artery of the brain (A. basilaris cerebri) in foxes. Folia Morphologica, Warszawa, v. 25, n. 4, p. 645-649, 1966

ZAGZAG, D.; BREM, S.; ROBERT, F. Neovascularization and tumor growth in the rabbit brain. A model for experimental studies of angiogenesis and the blood-brain barrier. American Journal of Pathology, Pennsylvania, v. 131, n. 2, p. 361-372, 1988. 